\title{
INFORME BIBLIOGRÁFICO: NIETZSCHE Y SCHOPENHAUER
}

AKL, Marie-Claude, «La recontre de Nietzsche et de Wagner dans la philosophie de Schopenhauer», Extasis. Bulletin du cercle philosophique, 1985, 23-35.

ANDOLFI, Ferruccio, «La responsabilidad de ser uno mismo: Schopenhauer, Nietzsche, Simmel», Debats, 57-58, 1996, 21-30.

ANGLET, Kurt, Zur Phantasmagorie der Tradition: Nietzsches Philosophie zwischen Historismus und Beschwörung: eine Studie auf der Grundlage der zweiten und dritten Unzeitgemäßen Betrachtung, Echter, Würzburg,1989.

AUSMUS, Harry J., A Schopenhauerian critique of Nietzsche's thought: toward a restoration of metaphysics, Mellen, Lewiston, 1996.

BAIG, Mirza Ahmad Ali, Nietzsche's Schopenhauer: the peak of modernity and the problem of affirmation, Chicago, Univ., Diss., 1994.

BANULS, André, «Schopenhauer und Nietzsche in Thomas Manns Frühwerk: Der Kleiderschrank und andere Novellen», Études Germaniques, 30, 1975, 129-147.

BARBERA, Sandro, «Ein Sinn und unzählige Hieroglyphen: einige Motive von Nietzsches Auseinandersetzung mit Schopenhauer in der Basler Zeit», en T. Borsche, F. Gerratana y A. Venturelli (eds), «Centauren-Geburten»: Wissenschaft, Kunst und Philosophie beim jungen Nietzsche, Gruyter, Berlin, 1994, 217-233.

_ , «Eine Quelle der frühen Schopenhauer-Kritik Nietzsches: Rudolf Hayms Aufsatz “Arthur Schopenhauer"», Nietzsche-Studien, 24, 1995, 124-136.

BARBOZA, Jair, «O eterno retorno do mesmo de Nietzsche na estética de Schopenhauer», Discurso, 28, São Paulo, 1997, 145-158.

BECKER, Frank, «Ästhetik als Korrektiv der Vernunft: Denkmotive Schopenhauers und Nietzsches in der ästhetischen Theorie Adornos», Zeitschrift für Ästhetik und allgemeine Kunstwissenschaft, 34, 1989, 28-50.

BERMAN, David, «Schopenhauer and Nietzsche: honest atheism, dishonest pessimism», en C. Janaway (ed.), Willing and nothingness: Schopenhauer as Nietzsche's educator, pp. 178-195.

BILSKER, Richard, Schopenhauer on willing and the unconscious: origins and legacy, Univ., Tallase, Diss., 1994.

BLONDEL, Eric, «Contre Kant et Schopenhauer: l'affirmation nietzscheénne», Revue philosophique de la France et de l'étranger, 123, 1998, 293-310.

BORNMANN, Fritz, «Epicuro nel pensiero di Schopenhauer e di Nietzsche», en V.F. Cicerone (ed.), Gli antichi e noi, Foggia, 1983, 29-44.

BURKE, James Vincent, Nietzsche's answer to Schopenhauer's pessimism: a study of its educational significance, Rutgers Univ., New Brunswick (NJ), Diss., 1976. 
BURGOS DIAZ, Elvira, «La presencia de Schopenhauer y Wagner en El nacimiento de la tragedia», Studium, 3, 1991, 219-229.

CABADA CASTRO, Manuel, «La reflexión sobre la negación o afirmación de la vida en Schopenhauer, Feuerbach, Wagner y Nietzsche», Pensamiento, 47,1991, 385-414.

- - Querer o no querer vivir: el debate entre Schopenhauer, Feuerbach, Wagner y Nietzsche sobre el sentido de la existencia humana, Herder, Barcelona, 1994.

CARTWRIGHT, David E., «Kant, Schopenhauer, and Nietzsche on the morality of pity», Journal of the history of ideas, 45, 1984, 83-98.

- , «Nietzsche's use and abuse of Schopenhauer's moral philosophy for life», en C. Janaway (ed), Willing and nothingness: Schopenhauer as Nietzsche's educator, pp. 116-150.

—_, «Reversing Silenus' wisdom», Nietzsche-Studien, 20, 1991, 309-313.

, «The last temptation of Zarathustra», Journal of the history of philosophy, 31, 1993, 49-69.

CASINI, Leonardo, La riscoperta del corpo: Schopenhauer / Feuerbach / Nietzsche, Edizioni Studium, Roma, 1990.

—_ "Mann interprete di Schopenhauer e di Nietzsche», Cultura tedesca = Deutsche Kultur. Rivista semestrale, 1, 1994, 51-60.

CASTILLO, Monique, «Entre Kant et Nietzsche: le destin des symboles», Les études philosophiques, 4, 1997, 433-451.

CAUCHI, Francesca, «Nietzsche and pessimism: the metaphysic hypostatised», History of European ideas, 13. 1991, 253-267.

CLARK, Maudemarie, «On knowledge, truth, and value: Nietzsche's debt to Schopenhauer and the development of his empiricism», en C. Janaway (ed), Willing and nothingness: Schopenhauer as Nietzsche's educator, pp. 37-78.

COOPER, David E., «Self and morality in Schopenhauer and Nietzsche», en C. Janaway (ed), Willing and nothingness: Schopenhauer as Nietzsche's educator, pp. 196-216.

DECHER, Friedhelm, «Der eine Wille und die vielen Willen: Schopenhauer Mainländer - Nietzsche», Nietzsche-Studien, 25, 1996, 221-238.

__ , «Nietzsches Metaphysik in der Geburt der Tragödie im Verhältnis zur Philosophie Schopenhauers», Nietzsche-Studien, 14, 1985. 110-125.

- Wille zum Leben Wille zur Macht: eine Untersuchung zu Schopenhauer und Nietzsche, Königshausen \& Neumann, Würzburg, 1984.

DElCARO, «Adrian, Denial versus affirmation: Kafka's «Ein Hungerkünstler» as a paradigm of freedom», Modern Austrian literature, 22, 1989. No. 1, 35-55.

D’HAUTEUILLE, François, «Schopenhauer, Nietzsche et Bergson», Archives de philosophie, 28, 1965, 553-566.

DIAS, Rosa Maria, «A influência de Schopenhauer na filosofia da arte de Nietzsche em $O$ nascimento da tragédia», Cadernos Nietzsche, 3, 1997, 7-21.

DUFOUR, Éric, «Métaphysique de la musique dans Le monde comme volonté et comme représentation et dans La naissance de la tragédie», Les études philosophiques, 1997, n. 4, 471-492.

DUVALL, Trumbull G., «Life is a task to be done, not understood - Schopenhauer and Nietzsche», en T.G. Duvall, Great thinkers, New York, 1937, 260-283. 
FAZIO, Domenico M., Nietzsche e il criticismo: elementi kantiani e neokantiani e critica della dialettica hegeliana nella formazione filosofica del giovane Nietzsche, QuattroVenti, Urbino, 1991.

FELLMANN, Ferdinand, Ethik der Selbsterfahrung nach Schopenhauer und Nietzsche, Münchner Volkshochschule, München, 1994.

FRANK, Erich, «Schopenhauer und Nietzsche», en E. Frank, Wissen, Wollen, Glauben. Knowledege, will and belief: gesammelte Aufsätze zur Philosophiegeschichte und Existentialphilosophie, Zürich/Stuttgart, 1955, 250-268.

GILMAN, Sander L., «Hegel, Schopenhauer and Nietzsche see the black», HegelStudien, 16, 1981, 163-188.

GOEDERT, Georges, Nietzsche der Überwinder Schopenhauers und des Mitleids, Königshausen \& Neumann, Würzburg 1988.

—_, «Nietzsche und Schopenhauer», Nietzsche-Studien, 7, 1978, 1-15.

, «Nietzsches Immoralismus, seine ambivalente Beziehung zu Schopenhauer», Schopenhauer-Jahrbuch, 65, 1984, 69-79.

_- «Über die Bedeutung der Beziehung zu Schopenhauer in Nietzsches Kritik der Demokratie», en W. Schirmacher (ed.), Schopenhauers Aktualität: ein Philosoph wird neu gelesen», Wien, 1988, 89-103.

HASSE, Heinrich, «Das Problem der Erlösung bei Schopenhauer und Nietzsche», Jahrbuch der Schopenhauer-Gesellschaft, 23, 1936, 100-120.

___ « « «orstufen der Lehre Nietzsches von der ewigen Wiederkunft bei Schopenhauer», Jahrbuch der Schopenhauer-Gesellschaft, 16, 1929, 45-56.

HEFTRICH, Urs, Otokar Bøezina: zur Rezeption Schopenhauers und Nietzsches im tschechischen Symbolismus, Winter, Heidelberg, 1993.

HIGGINS, Kathleen Marie, «Schopenhauer and Nietzsche: temperament and temporality», en C. Janaway (ed.), Willing and nothingness: Schopenhauer as Nietzsche's educator, pp. 151-177.

HILL, Randolph Kevin, Language, mind and reality : Nietzsche's overcoming of metaphysics, Univ. of Illinois, Urbana-Champaign, Diss., 1992.

HILLESHEIM, James Walter, The doctrine of self-perfection in Nietzsche's Schopenhauer as educateur, Tulane Univ. of Louisiana, Diss., 1964.

HÜBSCHER, Arthur, «Schopenhauer, Wagner, Nietzsche», Schopenhauer-Jahrbuch für das Jahr 1978, 59, 1978, 89-99.

INGENKAMP, Heinz Gerd, «Menschenliebe bei Schopenhauer und Nietzsche», en D. Birnbacher (ed), Schopenhauer in der Philosophie der Gegenwart, Königshausen \& Neumann, Würzburg, 1996, pp. 138 -158

JANAWAY, Christopher, «Nietzsche, the self, and the Schopenhauer», en K. AnsellPearson (ed.), Nietzsche and modern German thought, London/New York, 1991, pp. 119-142.

— nothingness: Schopenhauer as Nietzsche's educator, pp. 13 - 36.

- Self and world in Schopenhauer's philosophy, Clarendon Press, Oxford, 1989. (ed.), Willing and nothingness: Schopenhauer as Nietzsche's educator, Clarendon Press, Oxford, 1998,

JANKOWSKI, Wolf-Günther, "'Wahrheit' und 'Irrtum' bei Schopenhauer und Nietzsche», Schopenhauer-Jahrbuch, 58, 1977, 59-69. 
KÄTZEL, Siegfried, «Zur Kontinuität bei der Analyse der philosophischen Auffassungen von Arthur Schopenhauer und Friedrich Nietzsche in der Geschichte der marxistischen Philosophie», Beiträge zur Kritik der bürgerlichen Philosophie und Gesellschaftstheorie, 7/8, 1987, 198-209.

KIENZEL, Ulrike, «“... das freiwillige Leiden der Wahrhaftigkeit”: zu den philosophischen Hintergründen des Bruchs zwischen Wagner und Nietzsche : eine Reknstruktion ihres Dialogs über den Pessimismus Schopenhauers», en T. Steiert (ed), «Der Fall Wagner»: Ursprünge und Folgen von Nietzsches WagnerKritik, Laaber, 1991, pp. 81-136.

KIOWSKI, Hellmuth, Das Mitleid in der Ethik Schopenhauers in Kontrastierung mit Nietzsches Moral der Vornehmheit, Basel, Univ., Diss., 1986.

KLUGKIST, Thomas, Glühende Konstruktionen: Thomas Manns «Tristan» und das 'Dreigestirn': Schopenhauer, Nietzsche und Wagner, Königshausen \& Neumann, Würzburg. 1995.

KUEHNEMUND, Richard, «Schopenhauer und Nietzsche über Leben und Kunst (1788-1948)», Monatshefte für deutschen Unterricht, deutsche Sprache und Literatur, 40/8, 1948. 443.

LAND, Nick, «Art as insurrection: the question of aesthetics in Kant, Schopenhauer, and Nietzsche», en K. Ansell-Pearson, Nietzsche and modern Geman thought, London/New York, 1991, 240-256.

LANDMANN, Michael, «Nietzsches Schopenhauer-Erlebnis», en Geist und Leben : varia Nietzscheana, Bonn, 1951, pp. 7-39.

LYPP, Bernhard, «Philosophie als Meditation des Lebens: Reflexionen über das Verhältnis Nietzsches zu Schopenhauer». Schopenhauer-Jahrbuch, 65, 1984, 5568.

MANN, Thomas, Schopenhauer, Nietzsche y Freud, tr. de Andrés Sánchez Pascual, Alianza, Madrid, 2000.

MARGREITER, Reinhard, «Allverneinung und Allbejahung: der Grund des Willens bei Schopenhauer und Nietzsche», Schopenhauer-Jahrbuch, 65, 1984, 103-115.

MIGOTTI, Mark Harding Cheyne, The early Nietzsche and the question of redemption, Yale Univ., Diss., Haven, Conn, 1991.

MILLER, Louis William, The revelation of genius: toward an interpretation of Nietzsche's early development, Princeton, NJ, Diss., 1994.

MIRANDA DE ALMEIDA, Rogério, «La finalité, la providence et le hasard selon Nietzsche», Revue des sciences religieuses, 71/1, 1997, 79-89.

MURGUIA, Adolfo, «Nota sobre Schopenhauer y Nietzsche», Stromata, 31, 1975, 335-340.

NABAIS, Nuno, «Schopenhauer, Wagner e o sublime: para uma arqueologio do lugar de Nietzsche na estética da pos-modernidade», en L. Ribeiro dos Santos (ed), Educação estética e utopia política : (Colóquio comemorativo dos 200 anos da publicação de Para a paz perpétua, de I. Kant, e de Cartas sobre a educação estética do ser humano, de F. Schiller (1795-1995)), Lisboa, 1996, pp. 277-317.

NEGRONI, Bruno, «Giorgio Colli als Leser Schopenhauers: eine kritische Lektüre seines Nach Nietzsche», Schopenhauer-Jahrbuch, 65, 1984, 241-246.

NELSON, Byron, «Wagner, Schopenhauer, and Nietzsche : on the value of human action», The Opera quarterly, 6/4, 1989, 24-32. 
NEUMANN, Michael, Unterwegs zu den Inseln des Scheins: Kunstbegriff und literarische Form in der Romantik von Novalis bis Nietzsche, Klostermann, Frankfurt a. M., 1991.

NEYMEYR, Barbara, Ästhetische Autonomie als Abnormität: kritische Analysen zu Schopenhauers Ästhetik im Horizont seiner Willensmetaphysik, de Gruyter, Berlin, 1996

— Nietzsches Auseinandersetzung mit Schopenhauer und Kant», Philosophisches Jahrbuch, 102, 1995, 225-248.

___ «Das Genie als Hermaphrodit? Schopenhauers ästhetische Fertilitätsmetaphorik und ihr Verhältnis zu Nietzsche», Zeitschrift für Ästhetik und allgemeine Kunstwissenschaft, 40/2, 1995, 199-217.

NUSSBAUM, Martha C., «The transfiguration of intoxication: Nietzsche, Schopenhauer, and Dionysus», Arion. Ser. 3, 1/2, 1990/91. No. 2, 75-11.

NUTT, Kathleen Ann Craig, Reason and its discontents: Schopenhauer's critique of Kant as the foundation of the philosophy of Nietzsche, Queen's Univ., Belfast, Diss., 1991.

OEHLER, Christoph, «Schopenhauers und Nietzsches Ästhetik als Ausgangspunkt des modernen Irrationalismus?», Schopenhauer-Jahrbuch, 65, 1984, 80-90.

PENZO, Giorgio, «Il problema della cultura in Nietzsche e il rapporto tra Schopenhauer e Nietzsche secondo A. Drews», en G. Penzo, Friedrich Nietzsche o la verità come problema, Bologna, 1984, pp. 313-324.

PERNIN SEGISSEMENT, Marie-José, Nietzsche et Schopenhauer: encore et toujours la prédestination, L'Harmattan, Paris, 1999

PIEPER, Thomas, Überwindung des Welt-Leids: Loerkes Lyrik im Spannungsfeld zwischen Nietzsche und Schopenhauer, Lang, Frankfurt am Main, 1992.

QUESADA, Julio, Un pensamiento intempestivo: ontología, estética y política en $F$. Nietzsche, Anthropos, Barcelona, 1988.

RETHY, Robert Aaron, An introduction to the problem of affirmation in Nietzsche's thought, Pennsylvania State Univ., Diss., 1980.

RIDEL, Manfred, «Ein Seitenstück zur Geburt der Tragödie: Nietzsches Abkehr von Schopenhauer und Wagner und seine Wende zur Philosophie», Nietzsche-Studien, 24, 1995, 45-61.

RIPPEL, Philipp, «Malerei als dionysische Magie: Picasso im Kontext von Schopenhauer und Nietzsche», Neuе Rundschau, 105/3, 1994. 137-148.

RODRIGUEZ GONZÁLEZ, Mariano, «Metafisica de la finalidad natural: su metamorfosis en la linea Kant - Schopenhauer - Nietzsche», Pensamiento, 50/198, 1994, 435-455.

ROOS, Richard, «Der Schopenhauerische Mensch und die Historie in Nietzsches Unzeitgemäßen Betrachtungen», Schopenhauer-Jahrbuch, 65, 1984, 31-43.

ROTHACKER, Erich, «Schopenhauer et Nietzsche», Poètes et penseurs, Paris, 1941, pp. 11-57.

SALAQUARDA, Jörg, «Leib bin ich ganz und gar...: zum 'dritten Weg' bei Schopenhauer und Nietzsche», Nietzscheforschung, 1, 1994, 37-50. 
, «Nietzsches Metaphysikkritik und ihre Vorbereitung durch Schopenhauer», G. Abel y J. Salaquarda (eds), Krisis der Metaphysik, Berlin/New York, 1989, 258-282.

—__ «Zur gegenseitigen Verdrängung von Schopenhauer und Nietzsche», Schopenhauer-Jahrbuch, 65, 1984, 13-30.

SCHIRMACHER, Wolfgang, «Konkurs der Vernunft als Lebenskrise: zur Aktualität von Schopenhauer und Nietzsche», Schopenhauer-Jahrbuch, 65, 1984, 7-12.

(ed), Schopenhauer, Nietzsche und die Kunst, Passagen, Wien, 1991.

SCHLECHTA, Karl, «Der junge Nietzsche und Schopenhauer», Jahrbuch der Schopenhauer-Gesellschaft, 26, 1939, 289-300.

SCHNITZLER, Michael Hubert, «Die Lehre vom Willen bei Schopenhauer und Nietzsche und ihre pädagogische Auswirkung», Vierteljahrsschrift für wissenschaftliche Pädagogik, 2, 1926, 47-96.

SCHRÖDER-AGUSTIN, Markus, «Décadence und Lebenswille: Tonio Kröger im Kontext von Schopenhauer, Wagner und Nietzsche», Wirkendes Wort, 48!2, 1998, 255-274.

SHAH, Sanjiv, «Pessimism, nihilism, and the Nietzschean response», Dialogue, 39/ 2-3, 1997, 56-62.

SIMMEL, Georg, Schopenhauer y Nietzsche, tr. F. Ayala, Anaconda, Buenos Aires, 1950.

SOLL, Ivan, «Schopenhauer, Nietzsche, and the redemption of life through art», en

C. Janaway, Willing and nothingness: Schopenhauer as Nietzsche's educator, pp. 78-115.

SOMMER, Andreas Urs, "“Wenn die Welt ein Ding ist, das besser nicht wäre, ei so ist ja auch das Denken des Philosophen, das ein Stück dieser Welt bildet, ein Denken, das besser nicht dächte": zur Karriere eines polemischen Argumentes gegen Schopenhauer», Schopenhauer-Jahrbuch, 77, 1996, 199-210.

SONDAG, Yves, Nietzsche, «Schopenhauer, l'ascétisme et la psychanalyse», Revue philosophique de la France et de l'étranger, 96/161, 1971, 347-359.

SPIERLING, Volker, «Nietzsche y Schopenhauer: una comparación», Enrahonar, 25, 1996, 21-39.

SPINELLO, Richard Arthur, Nietzsche's conception of the body, Fordham Univ., Bronx, NY Diss., 1981.

SUANCES MARCOS, Manuel y VILLAR EZCURRA, Alicia, El irracionalismo, Síntesis, Madrid, 2000.

TAYLOR, Charles Senn, «Nietzsches's Schopenhauerianism», Nietzsche-Studien, 17, 1988.

TÖPFER, Hellmuth, Deutung und Wertung der Kunst bei Schopenhauer und Nietzsche, Dresden, Risse-Verlag, 1933.

WACHENDORFF, Elke-A., Das neue Denken der «Noth-Wendigkeit»: Leiden, Schmerz und Lust in der Philosophie Friedrich Nietzsches, Müchen, Univ., Diss., 1991.

WEIMER, Wolfgang, «Die ewige Wiederkehr des Gleichen bei Schopenhauer und Nietzsche», Schopenhauer-Jahrbuch, 65, 1984, 44-54.

WELCH, Gregory Stephen, Der Schopenhauer Fall: Schopenhauer as Nietzsches educator, Cambridge, Mass., Harvard Univ., Diss., 1990. 
WIELE, Jozef van de, «Schopenhauer et le volontarisme: aux sources de Nietzsche», Revue philosophique de Louvain, 74, 1976, 375-400.

WINKLER, Gerhard J., «Der 'Schleier der Maja': Transformationen eines Begriffs Schopenhauer - Wagner - Nietzsche», en T. Steiert, «Der Fall Wagner»: Ursprünge und Folgen von Nietzsches Wagner-Kritik, Laaber, 1991, pp. 233263.

WISCHKE, Mirko, Die Geburt der Ethik: Schopenhauer, Nietzsche, Adorno. Akademie-Verlag, Berlin, 1994.

YOUNIS, Raymond Aaron, «"A cold eye of life, on death": Schopenhauer, Nietzsche and Yeats on "passing by"», English language notes, 30/2, 1992, 50-56.

ZINT, Hans, «Schopenhauer als Erlebnis», Jahrbuch der Schopenhauer-Gesellschaft, 25, 1938, pp. 92-130.

elaborado por Luis E. De Santiago Guervós 